\title{
Capacity Analysis of Distributed Antenna Systems with Beamforming
}

\author{
Meiyan Ju, Chao Chen, Yueheng Li and Guoping Tan \\ College of Computer and Information, Hohai University, Nanjing 211100, China
}

\begin{abstract}
Distributed antenna systems (DASs) can reduce the access distance, increase system capacity and improve system performance compared with conventional centralized multiple antenna systems. In this paper, the system capacity of downlink DAS with beamforming is investigated. The mathematical expression of system capacity is derived over the composite channel including Rayleigh fading, shadowing and path loss. At the same time, the interference from other APs is also considered for the studied AP. Numerical methods including Gauss-Hermite integral and composite Simpson's rule are used to compute the system capacity of DAS under different MS distributions. Numerical results verify the precision of the mathematical analysis of system capacity by comparing Monte-Carlo simulation results. The results also show interference among APs shouldn't be ignored when APs are close to each other or there are many APs.
\end{abstract}

\section{Introduction}

A distributed antenna system (DAS), which has multiple antenna ports (APs) in each cell, has attracted wide attention recently because it can increase coverage, reduce transmit power and improve the sum rate and cell-edge performance in cellular communication systems $[1,2,3]$.

Many efforts have been devoted to analyze the performance of DAS with random or fixed antenna layout. System capacity is an important performance indicator in wireless communication systems. Capacity performance and antenna placement design of DAS in different scenarios were studied well in $[4,5,6]$, but the previous studies rarely discuss the capacity of DAS with beamforming. [7] derived the optimal beamforming vector of downlink DAS with a single user at the fixed location and also analyzed the outage probability and ergodic capacity. However, it didn't consider the random location of the user. [8] studied the optimal AP distribution to make the sum of user rates under all APs transmitting data in a single-cell downlink DAS with beamforming over a simple composite channel. However, [8] only considered the channel including rapid fading and path loss and the scenario that there was only a single antenna at each AP and mobile terminal. Although $[7,8]$ studied the DAS with beamforming, they didn't consider and analyze the interference among APs. In practical communication systems, the interference among APs exists. Compared with the interference from APs of other cells, the interference from other APs in the same cell should not be neglected for some studied AP.

In this paper, based on what we have studied in [9], system capacity of downlink DAS with beamforming have been studied here for two scenarios including neglecting the interference from other APs and considering the interference from other APs. First, based on the composite channel model including path loss, shadow fading and Rayleigh fading, a DAS model with beamforming is built. Then for the DAS with one AP in the center, the mathematical expressions of system capacity are derived under neglecting interference among APs and considering interference among APs. Furthermore, the expressions are computed by numerical methods. Considering uniform distribution and quasiuniform distribution of MS position, the numerical results verify the correctness and precision of the theoretical analysis and show that the interference among APs has an impact on system capacity, especially when APs are close to each other and the number of APs is not small.

The rest of the paper is organized as follows. Section 2 shows the system model of DAS with beamforming. The system capacity performance analysis is presented in Section 3. Simulation results are shown in Section 4. Section 5 concludes the paper.

Throughout this paper, we employ bold upper and lower case letters for matrices and vectors respectively. The superscript $(\cdot)^{T}$ and $(\cdot)^{*}$ denote transpose and conjugate respectively. $\mid \cdot$ and \|\|$^{2}$ stand for modulus (magnitude) and Frobenius norm respectively. The expectation of a random variable is given by $E(\cdot)$.

\section{System model}

In this section, we consider a downlink DAS with a single cell where $M$ APs with $N$ antennas communicate to a mobile user with a single antenna, as

\footnotetext{
* Corresponding author: ${ }^{\text {a }}$ Meiyan Ju: jmy@hhu.edu.cn
} 
shown in Fig. 1. Assuming all APs use the same frequency resource to communicate, all APs, which are denoted by $\mathrm{AP}_{i}(i=0,1, \cdots, M-1)$, are connected to MPU to communicate information. In our system, one $\mathrm{AP}$ is at the center of the cell and the other APs are distributed at different locations of the same circle. Without loss of generality, we use the polar coordinates $(\rho, \theta)$ and $\left(\rho_{i}, \theta_{i}\right)$ to denote the positions of the MS and $\mathrm{AP}_{i}$. Here $(\rho, \theta)$ and $\left(\rho_{i}, \theta_{i}\right)$ are the distances and angles relative to the cell center. Due to the large space among APs in DAS, the channel model between a AP and a MS involves both small scale fadings and large scale fadings including shadowing and path loss.

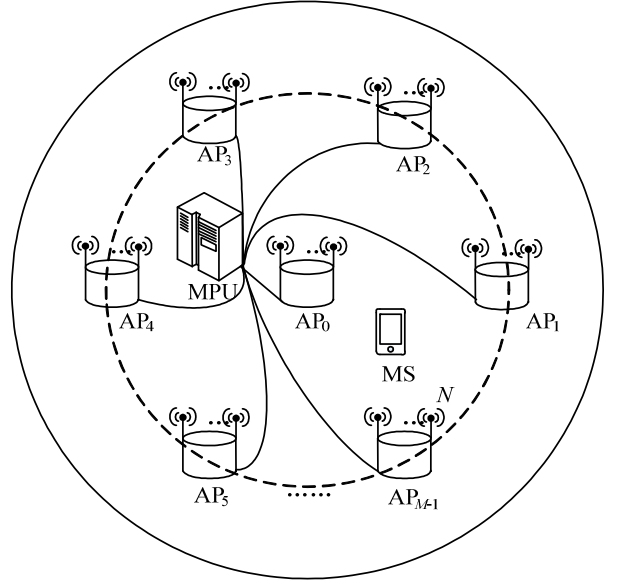

Figure 1. The system structure of DAS in a single cell

Now the downlink transmission of DAS is considered, and the received signal at a $\mathrm{MS}$ from $\mathrm{AP}_{i}$ can be expressed as

$$
y_{i}=\sqrt{P} \boldsymbol{h}_{i}^{T} \boldsymbol{w}_{i} x+z
$$

where $P$ denotes the transmit signal power, $\boldsymbol{w}_{i}$ is the beamforming vector at the transmitter, $x$ represents the transmitted symbol with unit energy, and $\boldsymbol{h}_{i}=\left[\begin{array}{llll}h_{i}^{(1)} & h_{i}^{(2)} & \cdots & h_{i}^{(N)}\end{array}\right]^{T}$ denotes the channel fading from all antennas of $\mathrm{AP}_{i}$ to the MS. $z$ stands for the complex Gaussian noise variable with zero mean and variance $N_{0}$.

Here the channel model includes path loss, shadowing and Rayleigh fading. $h_{i}^{(n)}$ can be mathematically expressed by

$$
h_{i}^{(n)}=g_{i}^{(n)} \sqrt{S_{i} L_{i}}
$$

where $S_{i}$ and $L_{i}$ are shadowing and path loss. $g_{i}^{(n)}$ represents the rapid fading from the $n$-th antenna of $\mathrm{AP}_{i}$ to the MS in the cell. Since different antennas of each AP are very close, all $h_{i}^{(n)}$ 's $(n=1,2, \cdots, N)$ have the same shadowing and path loss. Here we assume path loss, shadowing, and the small scale fading are independent. Assuming $g_{i}^{(n)}$ is Rayleigh fading, we have $\left|g_{i}^{(n)}\right|^{2}$ conforming to exponential distribution [10]. If the mean of $\left|g_{i}^{(n)}\right|^{2}$ is unit, its probability density function (pdf) can be written by

$$
f_{\left|g_{i}^{(n)}\right|^{2}}(x)=\exp (-x), \quad x \geq 0 .
$$

Path loss, $L_{i}$ in (2), can be denoted as

$$
L_{i}=\left(\frac{d_{0}}{d_{i}}\right)^{\alpha_{i}}
$$

where $\alpha_{i}$ is the path loss exponent of $\mathrm{AP}_{i}, d_{0}$ denotes the reference distance, and $d_{i}$ represents the distance between $\mathrm{AP}_{i}$ placed at $\left(\rho_{i}, \theta_{i}\right)$ and the MS located at $(\rho, \theta)$, which can be given by

$$
d_{i}(\rho, \theta)=\sqrt{\rho^{2}+\rho_{i}^{2}-2 \rho \rho_{i} \cos \left(\theta-\theta_{i}\right)} .
$$

Here $d_{i}=d_{0}$ is set when $d_{i}<d_{0}$. Shadowing $S_{i}$ is a random variable with lognormal distribution. Given the mean and the standard derivation of $10 \log _{10} S_{i}$ are $0 \mathrm{~dB}$ and $\sigma_{i} \mathrm{~dB}$ respectively, the pdf of $S_{i}$ is given as

$$
f_{S_{i}}(s)=\frac{\xi}{\sqrt{2 \pi} \sigma_{i} s} \exp \left[-\frac{\left(10 \log _{10} s\right)^{2}}{2 \sigma_{i}^{2}}\right], \quad s>0
$$

where $\xi=10 / \ln 10$.

\section{System capacity analysis}

The system capacity is an important index to evaluate the system performance. The basis of capacity is to analyze the signal-to-noise ratio (SNR). We assume the channel state information between $\mathrm{AP}_{i}$ and $\mathrm{MS}$ is known and selective transmission (ST) is chosen, which means the AP that makes MS get the maximal receive SNR is chosen to transmit information. The SNR of downlink DAS with beamforming is analyzed under two scenarios including each AP free from interference of other APs and each AP receiving interference of other APs, based on which the system capacity is further discussed.

\subsection{Signal-to-noise ratio analysis without interference from other APs}

We use the optimal beamforming vector at $\mathrm{AP}_{i}$, which is expressed as $\boldsymbol{w}_{i}=\frac{\boldsymbol{h}_{i}^{*}}{\left\|\boldsymbol{h}_{i}\right\|}[7,11]$. Using (1) and (2), the received SNR for the MS located at $(\rho, \theta)$ is expressed as

$$
\gamma_{i}=\frac{P\left|\boldsymbol{h}_{i}^{T} \boldsymbol{w}_{i}\right|^{2}}{N_{0}}=\frac{P\left\|\boldsymbol{h}_{i}\right\|^{2}}{N_{0}}=\sum_{n=1}^{N} \Omega_{i}\left|g_{i}^{(n)}\right|^{2}
$$

where $\Omega_{i}=\frac{P}{N_{0}} S_{i} L_{i}$. Since $L_{i}$ is path loss related to the distance and $S_{i}$ is a lognormal random variable, $\Omega_{i}$ is also a lognormal random variable. The mean and standard derivation of $10 \log _{10} \Omega_{i}$ are denoted as 
$\mu_{i}(\rho, \theta)=10 \log _{10}\left(P / N_{0}\right)+10 \log _{10}\left(L_{i}\right)$ and $\sigma_{i}$. Then we obtain the pdf of $\Omega_{i}$

$$
f_{\Omega_{i}}(\Omega)=\frac{\xi}{\sqrt{2 \pi} \sigma_{i} \Omega} \exp \left[-\frac{\left(10 \log _{10} \Omega-\mu_{i}(\rho, \theta)\right)^{2}}{2 \sigma_{i}^{2}}\right] \text {. (8) }
$$

Assuming $g_{i}^{(1)}, g_{i}^{(2)}, \cdots, g_{i}^{(n)}$ are independent with each other, the conditional moment generating function [10] can be written as

$$
M_{\Upsilon_{i} \mid \Omega_{i}}(p \mid \Omega)=(1-p \Omega)^{-N} .
$$

Then we obtain the conditional pdf

$$
f_{\gamma_{i} \mid \Omega_{i}}(\gamma \mid \Omega)=\ell^{-1}\left[M_{\gamma_{i} \mid \Omega_{i}}(-p \mid \Omega)\right]=\frac{\gamma^{N-1}}{\Omega^{N} \Gamma(N)} \exp \left(-\frac{\gamma}{\Omega}\right)
$$

where $\ell^{-1}(\cdot)$ represents inverse Laplace transformation, and $\Gamma(\cdot)$ denotes gamma function.

Combining (8) and (10), we can derive the pdf of the received SNR $\gamma_{i}$

$$
\begin{aligned}
& f_{\gamma_{i}}(\gamma)=\int_{0}^{+\infty} f_{\gamma_{i} \mid \Omega_{i}}(\gamma \mid \Omega) f_{\Omega_{i}}(\Omega) d \Omega \\
& =\int_{0}^{+\infty} \frac{\gamma^{N-1}}{\Omega^{N} \Gamma(N)} \exp \left(-\frac{\gamma}{\Omega}\right) \\
& \times \frac{\xi}{\sqrt{2 \pi} \sigma_{i} \Omega} \exp \left[-\frac{\left(10 \log _{10} \Omega-\mu_{i}(\rho, \theta)\right)^{2}}{2 \sigma_{i}^{2}}\right] d \Omega .
\end{aligned}
$$

\subsection{SNR analysis with interference from other APs}

Assuming all the APs have the same transmit power, here we consider the interference from other APs to $\mathrm{AP}_{i}$ in the same cell. The received signal at MS given by (1) is transformed into

$$
y_{i}=\sqrt{P} \boldsymbol{h}_{i}^{T} \boldsymbol{w}_{i} x_{i}+\sum_{\substack{j=0 \\ j \neq i}}^{M-1} \sqrt{P} \boldsymbol{h}_{j}^{T} \boldsymbol{w}_{j} x_{j}+z=\sqrt{P} \boldsymbol{h}_{i}^{T} \boldsymbol{w}_{i} x_{i}+z^{\prime}
$$

where $x_{i}$ is the normalized signal transmitted by $\mathrm{AP}_{i}$, and $x_{j}$ is the normalized signal from $\mathrm{AP}_{j}(j \neq i)$. Likewise, each $\operatorname{AP}_{i}(i=0,1, \cdots, M-1)$ adopts the optimal beamforming vector $\boldsymbol{w}_{i}=\frac{\boldsymbol{h}_{i}^{*}}{\left\|\boldsymbol{h}_{i}\right\|}$. The interference from other APs can be taken as part of noise. $z^{\prime}$ is the equivalent noise including the noise and the interference from other APs. The received SNR for the MS located at $(\rho, \theta)$ is given by

$$
\gamma_{i}=\frac{P\left|\boldsymbol{h}_{i}^{T} \boldsymbol{w}_{i}\right|^{2}}{\sigma_{n}^{2}}=\frac{P\left\|\boldsymbol{h}_{i}\right\|^{2}}{P \sum_{\substack{j=0 \\ j \neq i}}^{M-1} N \cdot S_{j} L_{j}+N_{0}}=\sum_{n=1}^{N} \Omega_{i}\left|g_{i}^{(n)}\right|^{2}
$$

where $\Omega_{i}=\frac{P}{\sigma_{n}^{2}} S_{i} L_{i}$ and $\sigma_{n}^{2}=P \sum_{\substack{j=0 \\ j \neq i}}^{M-1} N \cdot S_{j} L_{j}+N_{0}$ which is the variance of $z^{\prime}$. The corresponding mathematical formulations from (8) to (11) in Section 3.1 can be applied here, too. Using them, we can derive the pdf of the received SNR after considering the interference from other APs. The only difference lies in $\Omega_{i}$.

\subsection{System capacity analysis}

In this section, assuming ST scheme is applied, the downlink system capacity of DAS is analyzed. The output SNR can be denoted as

$$
\gamma=\max \left\{\gamma_{0}, \gamma_{1}, \cdots, \gamma_{M-1}\right\} \text {. }
$$

Since distributed APs are sufficiently separated from each other, all the links between antennas of APs and the MS are assumed to undergo independent fading. Therefore, $\gamma_{i}$ in (14) is independent of $\gamma_{j}(i \neq j)$.

According to Shannon equation, the average capacity can be denoted as

$$
C=\int_{0}^{\infty} \log _{2}(1+\gamma) f(\gamma) \mathrm{d} \gamma
$$

where $\gamma$ denotes SNR value and $f(\gamma)$ is the pdf of SNR.

First, we need to analyze the cumulative distribution function (cdf) of $\gamma_{i}$. For (11), let $\Omega^{\prime}=N \Omega$, it is derived as

$$
\begin{aligned}
& f_{\gamma_{i}}(\gamma)=\int_{0}^{+\infty} \frac{N^{N} \gamma^{N-1}}{\Omega^{\prime N} \Gamma(N)} \exp \left(-\frac{N \gamma}{\Omega^{\prime}}\right) \frac{\xi}{\sqrt{2 \pi} \sigma_{i} \Omega^{\prime}} \\
& \times \exp \left[-\frac{\left(10 \log _{10} \Omega^{\prime}-10 \log _{10} N-\mu_{i}(\rho, \theta)\right)^{2}}{2 \sigma_{i}^{2}}\right] d \Omega^{\prime}
\end{aligned}
$$

From (16), we can see that the received SNR is conformed to Gamma-Lognormal distribution. Since [12] showed that lognormal distribution can approximate Gamma-Lognormal distribution well, the pdf in (16) can be rewritten as

$$
f_{\gamma_{i}}(\gamma) \approx \frac{\xi}{\sqrt{2 \pi} \hat{\sigma}_{i} \gamma} \exp \left[-\frac{\left(10 \lg \gamma-\hat{\mu}_{i}\right)^{2}}{2 \hat{\sigma}_{i}^{2}}\right], \gamma \geq 0
$$

where $\hat{\mu}_{i}$ and $\hat{\sigma}_{i}$ are the mean and variance of $10 \lg \gamma$, which use $\mathrm{dB}$ as unit. The values of $\hat{\mu}_{i}$ and $\hat{\sigma}_{i}$ are derived by the pdf in (16). Compute the expectation of $\gamma$ to get $\hat{\mu}_{i}$ as

$$
\begin{aligned}
\hat{\mu}_{i} & =E[10 \lg (\gamma)]=\int_{0}^{\infty} 10 \lg (\gamma) f_{\gamma_{i}}(\gamma) \mathrm{d} \gamma \\
& =\mu_{i}+\xi[\psi(N)-\ln (N)]
\end{aligned}
$$

where $\psi(v)$ is Digamma function, which is defined as

$$
\psi(z)=\mathrm{d}[\ln \Gamma(z)] / \mathrm{d} z=\Gamma^{\prime}(z) / \Gamma(z) .
$$

According to the definition of variance, we can get 


$$
\begin{aligned}
& \hat{\sigma}_{i}^{2}=E\left\{[10 \lg (\gamma)]^{2}\right\}-\{E[10 \lg (\gamma)]\}^{2} \\
& =\int_{0}^{\infty}[10 \lg (\gamma)]^{2} f_{\gamma_{i}}(\gamma) \mathrm{d} \gamma-\hat{\mu}_{i}^{2}=\sigma_{i}^{2}+\xi^{2} \zeta(2, N)
\end{aligned}
$$

where $\zeta(\cdot, \cdot)$ is Riemann zeta function [13], which is defined as

$$
\zeta(2, N)=\sum_{k=0}^{\infty} \frac{1}{(N+k)^{2}} .
$$

Substitute (18) and (20) into (17), we can get the pdf of receive SNR $\gamma_{i}$ at the MS from antenna port $\mathrm{AP}_{i}$. Further the cdf of $\gamma_{i}$ is derived as

$$
F_{\gamma_{i}}(x)=\int_{0}^{x} f_{\gamma_{i}}(\gamma) \mathrm{d} \gamma=1-\frac{1}{2} \operatorname{erfc}\left(\frac{10 \lg x-\hat{\mu}_{i}}{\sqrt{2} \hat{\sigma}_{i}}\right)
$$

where $\operatorname{erfc}(\cdot)$ is complementary error function, which is defined as

$$
\operatorname{erfc}(x)=\frac{2}{\sqrt{\pi}} \int_{x}^{\infty} e^{-t^{2}} d t
$$

The receive SNR values from different APs are independent with each other. Since selective transmission is chosen, the output SNR is the maximal one of all receive SNR values. Accordingly, the cdf of system output SNR is

$$
\begin{aligned}
& F_{\gamma}(x)=\operatorname{Pr}(\gamma<x)=\prod_{i=0}^{M-1} \operatorname{Pr}\left(\gamma_{i}<x\right)=\prod_{i=0}^{M-1} F_{\gamma_{i}}(x) \\
& =\prod_{i=0}^{M-1}\left[1-\frac{1}{2} \operatorname{erfc}\left(\frac{10 \lg x-\hat{\mu}_{i}}{\sqrt{2} \hat{\sigma}_{i}}\right)\right] .
\end{aligned}
$$

We compute the derivation of cdf in (24) and get the pdf of system output SNR

$$
\begin{aligned}
& f_{\gamma}(x)=F_{\gamma}{ }^{\prime}(x)=\sum_{i=0}^{M-1}\left[f_{\gamma_{i}}(x) \prod_{\substack{j=0 \\
j \neq i}}^{M-1} F_{\gamma_{j}}(x)\right] \\
= & \sum_{i=0}^{M-1}\left\{\frac{\xi}{\sqrt{2 \pi} \hat{\sigma}_{i} x} e^{-\frac{\left(10 \lg x-\hat{\mu}_{i}\right)^{2}}{2 \hat{\sigma}_{i}^{2}}} \prod_{\substack{j=0 \\
j \neq i}}^{M-1}\left[1-\frac{1}{2} \operatorname{erfc}\left(\frac{10 \lg x-\hat{\mu}_{j}}{\sqrt{2} \hat{\sigma}_{j}}\right)\right]\right\}
\end{aligned}
$$

where $f_{\gamma_{i}}(x)$ is as (17). Substituting (25) into (15), the obtained system capacity expression is complex infinite integral and we cannot get the numerical solution directly. To solve this problem, using Gauss-Hermite integral equation [13] to simplify it, let $r=\left(10 \lg x-\hat{\mu}_{i}\right) /\left(\sqrt{2} \hat{\sigma}_{i}\right)$, we get

$C=\int_{0}^{\infty} \log _{2}(1+x) f_{\gamma}(x) \mathrm{d} x$

$=\sum_{i=0}^{M-1} \int_{-\infty}^{+\infty} \log _{2}\left(1+10^{\frac{\sqrt{2} \hat{\sigma}_{i}+\hat{\mu}_{i}}{10}}\right) \frac{e^{-r^{2}}}{\sqrt{\pi}} \prod_{\substack{j=0 \\ j \neq i}}^{M-1}\left[1-\frac{1}{2} \operatorname{erfc}\left(\frac{\sqrt{2} \hat{\sigma}_{i} r+\hat{\mu}_{i}-\hat{\mu}_{j}}{\sqrt{2} \hat{\sigma}_{j}}\right)\right] \mathrm{d} r$

$$
\simeq \sum_{i=0}^{M-1} \sum_{k=1}^{K}\left\{\frac{H_{k}}{\sqrt{\pi}} \log _{2}\left(1+10^{\frac{\sqrt{2} \hat{\sigma}_{i} t_{t}+\hat{\mu}_{i}}{10}}\right) \prod_{\substack{j=0 \\ j \neq i}}^{M-1}\left[1-\frac{1}{2} \operatorname{erfc}\left(\frac{\sqrt{2} \hat{\sigma}_{i} t_{k}+\hat{\mu}_{i}-\hat{\mu}_{j}}{\sqrt{2} \hat{\sigma}_{j}}\right)\right]\right\}
$$

In (26), $t_{k}$ and $H_{k}$ are respectively the base point and weight factor of $K$-order Hermite polynomial. $\hat{\mu}_{i}$ and $\hat{\mu}_{j}$ are functions of the position of MS $(\rho, \theta)$. Since the capacity is related to the MS position, we denote the capacity in (26) as $C(\rho, \theta)$. To compute the average value of capacity for different MSs located in the cell, the average system capacity is

$$
\bar{C}=E[C(\rho, \theta)]=\int_{0}^{R} \int_{0}^{2 \pi} C(\rho, \theta) f(\rho, \theta) \mathrm{d} \rho \mathrm{d} \theta
$$

where $f(\rho, \theta)$ is the pdf of MS and may be very complex. The expression of $\bar{C}$ in (27) is not easy to compute the integral.

Via the two-dimensional composite Simpson's rule [14], the final system capacity can be approximated as

$$
\bar{C} \cong \frac{h q}{9} \sum_{b=0}^{B} \sum_{c=0}^{C}\left[a_{b, c} C\left(\rho_{b}, \theta_{c}\right) f\left(\rho_{b}, \theta_{c}\right)\right]
$$

where $B$ and $C$ are even numbers. The values of the step sizes $h$ and $q$ are chosen as $h=R / B$ and $q=2 \pi / C$. Then the values of $\rho_{b}$ and $\theta_{c}$ are $\rho_{b}=b h$, $(b=0,1, \cdots, B)$ and $\theta_{c}=c q,(c=0,1, \cdots, C)$. The weight factor $a_{b, c}$ is the element of the $(b+1)$-th row and $(c+1)$-th colomn in matrix $\boldsymbol{A}$, which is given by $\boldsymbol{A}=\underbrace{\left[\begin{array}{llllllll}1 & 4 & 2 & 4 & \cdots & 2 & 4 & 1\end{array}\right]^{T}}_{B+1} \underbrace{\left[\begin{array}{llllllll}1 & 4 & 2 & 4 & \cdots & 2 & 4 & 1\end{array}\right]}$.

Substituting (26) into (28), we can get the approximate analytical expression of the average system capacity.

\section{Numerical results}

In this section, the capacity of a single-cell DAS with beamforming is simulated. Different MS distributions and interference among APs are taken into account. One $\mathrm{AP}$ is at the cell center and the others are located uniformly at the same circle with the location $\left(\rho_{i}, \theta_{i}\right)$. It is assumed that all APs have the same transmit signal power. The main parameters used in simulations are listed in Table 1.

Table 1. Main simulation parameters.

\begin{tabular}{|c|c|}
\hline Parameter & Values \\
\hline$R$ & $1000 \mathrm{~m}$ \\
\hline$M$ & 7 \\
\hline$N$ & 10 \\
\hline$d_{0}$ & $40 \mathrm{~m}$ \\
\hline$\sigma_{\mathrm{i}}$ & $6 \mathrm{~dB}$ \\
\hline
\end{tabular}




\begin{tabular}{|c|c|}
\hline$\alpha_{\mathrm{i}}$ & 4 \\
\hline$K$ & 10 \\
\hline$B$ & 20 \\
\hline$C$ & 20 \\
\hline$\left(\rho_{0}, \theta_{0}\right)$ & $(0,0)$ \\
\hline
\end{tabular}

Here we consider two kinds of MS distribution. One is called uniform distribution, whose joint pdf in Cartesian coordinates is given as

$$
f(x, y)=\frac{1}{\pi R^{2}}
$$

where $R$ is the cell radius. Due to the relation between polar coordinates and Cartesian coordinates, we can obtain

$$
f(\rho, \theta)=\frac{\rho}{\pi R^{2}} .
$$

The other distribution of MS is quasi-uniform [15], and the joint pdf about $\rho$ and $\theta$ can be derived as

$$
f(\rho, \theta)=\frac{1}{2 \pi R} .
$$

Fig. 2 is the system capacity of DAS versus the transmit SNR $\left(P / N_{0}\right)$ under different MS distribution and interference scenarios when $\rho_{i}=0.6 R$ and $\theta_{i}=2 \pi i /(M-1)(i=1,2, \cdots, M-1)$. We can see that the simulation results by Monte-Carlo method are very close to the numerical results of theoretical expressions, which proves the correctness of the above derived theoretical formulations of system performance. It also shows that the system capacity improves as the value of transmit SNR increases. It is seen that the capacity of quasiuniform MS distribution is bigger than that of uniform MS distribution under the same interference scenarios. When the interference among APs is considered, the system capacity degrades and the gap becomes more obvious with increasing SNR under the same MS distribution. That is because the interference from other APs becomes more serious and the performance loss increases when transmit SNR values of APs are increasing.

Fig. 3 is the system capacity of DAS versus normalized distance of APs $\left(\rho_{i} / R\right)$ under different MS distribution and interference scenarios when transmit SNR is $30 \mathrm{~dB}$. From Fig. 3, it can be seen that the optimal normalized distance is different under different scenarios. In addition, the system capacity has a big difference between neglecting interference among APs and considering interference among APs for the same MS distribution when the normalized distance is less than 0.7. The gap becomes bigger when the normalized distance is smaller. That's because the APs are closer to each other when the normalized distance is getting smaller, which leads to the severe interference and makes the capacity performance degrade. For this situation, the interference among APs cannot be neglected. When APs are far from each other, such as the normalized distance is close to 1 , the interference from other APs decreases and will not have a big effect on system capacity, which leads to the small performance gap between considering interference and neglecting interference. Furthermore, the performance gap under quasi-uniform distribution is bigger than that under uniform distribution.

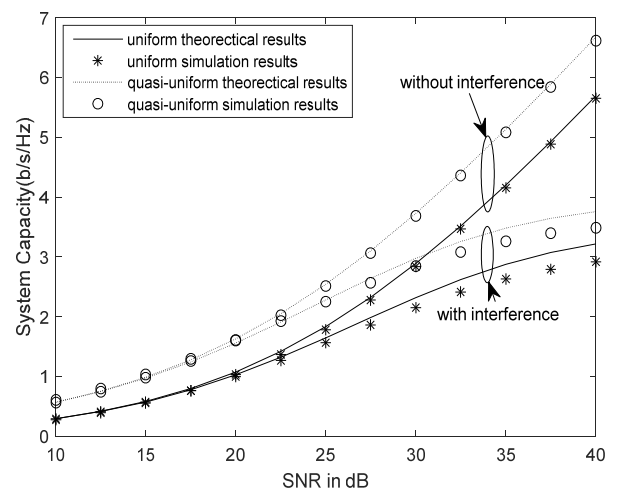

Figure 2. System capacity vs. SNR under different scenarios

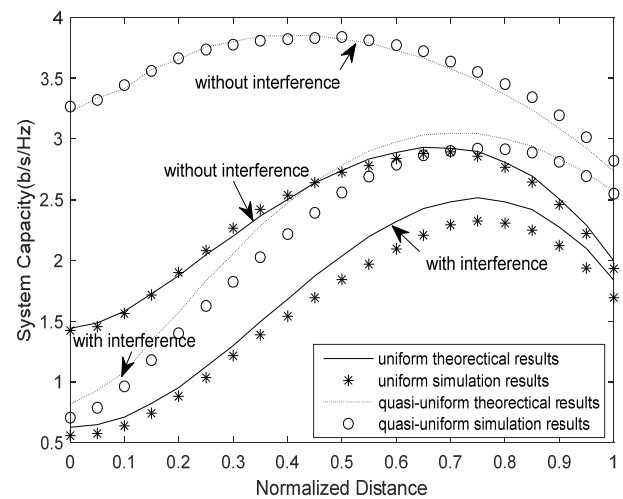

Figure 3. System capacity vs. normalized distance under different scenarios.

Fig. 4 and Fig. 5 are the system capacity performances for $M=4,7$, and 10 when $\rho_{i}=0.6 R$ and $\theta_{i}=2 \pi i /(M-1) \quad(i=1,2, \cdots, M-1) \quad$ under neglecting interference among APs and considering interference among APs respectively. From Fig. 4, it can be seen that the capacity is becoming bigger with the increasing number of APs for the same MS distribution when there is no interference from other APs. That is because the DAS can acquire more diversity gain from the increasing APs when the interference isn't considered. Fig. 5 shows the capacity performance doesn't keep getting better with increasing APs when the interference among APs is considered. From Fig. 5, When the SNR value is fairly big, the performance doesn't increase much with increasing SNR and is even worse than that of DAS with less APs. That may be because the interference is more serious when there are more APs. From Fig. $4 \sim$ Fig. 5, it can be seen that the relationship between the system performance and the number of APs changes after the interference among APs is considered. 


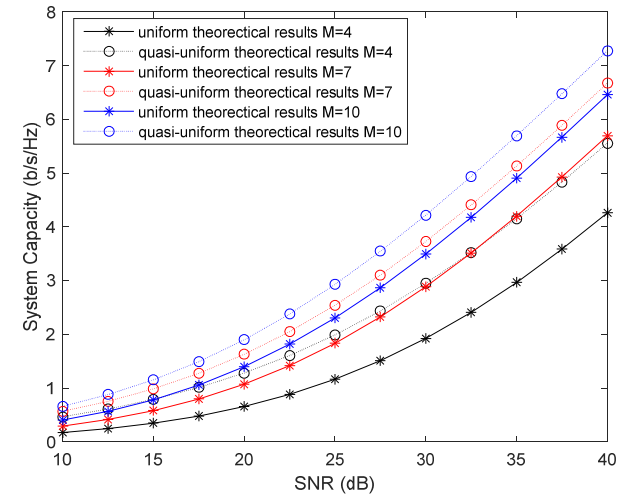

Figure 4. System capacity vs. SNR for different numbers of APs neglecting interference.

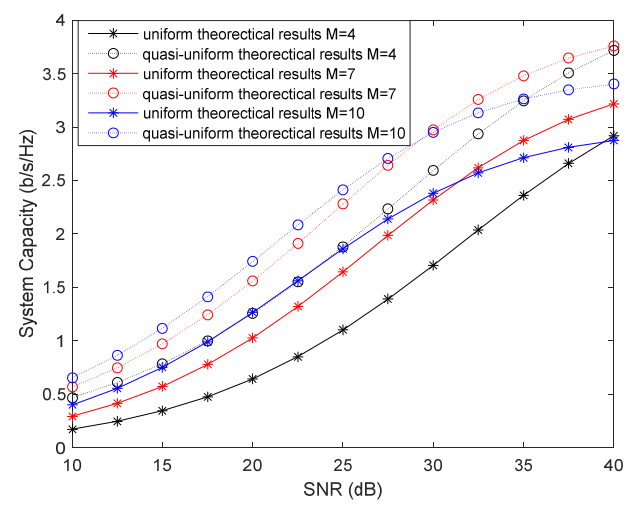

Figure 5. System capacity vs. SNR for different numbers of APs considering interference.

\section{Conclusions}

This paper discusses the downlink system capacity of DAS with beamforming in a single cell. Based on a composite channel including large scale fading and small scale fading, using Lognormal approximation to complicated Gamma-Lognormal distribution and numerical methods, the closed-form expressions of the system capacity are derived under two scenarios including neglecting and considering interference among APs. The numerical results prove that the theoretical analysis of system capacity is precise and also show that different MS distributions and interference among APs will have an effect on system capacity.

\section{References}

1. W. Roh, High performance distributied antenna cellular networks, Ph.D. dissertation, Dept. Elect. Eng., Stanford Univ., Stanford, CA (2003)

2. Cong Shen, Zehao $\mathrm{Yu}$, Changwen Chen, and Feng $\mathrm{Wu}$, On the effective capacities of distributed and co-located large-scale antenna systems, In Proc. of IEEE International Conference on Communications - Communication Theory Symposium, 1-6 (2017)

3. Jiamin Li, Dongming Wang, Pengcheng Zhu, Jiangzhou Wang, and Xiaohu You, Downlink spectral efficiency of distributed massive
MIMO systems with linear beamforming under Pilot Contamination, IEEE Transactions on Vehicular Technology, 67, 2, 1130-1145 (2018)

4. X. Wang, P. Zhu, and M. Chen, Antenna location optimization for circular-layout distributed antenna systems, IEICE Trans. on Com., E92-B, 9, 29802983 (2009)

5. Jin-Yuan Wang, Jun-Bo Wang, and Ming Chen, System capacity analysis and antenna placement optimization for downlink transmission in distributed antenna systems, Wireless Pers. Commun., 1-24 (2012)

6. Hongrui Wang, Dongming Wang, Ruixun Liu, Hongxiang Xie, and Zhaohui Yang, Antenna location design for distributed antenna systems with pilot contamination, International Conference on Wireless Communications and Signal Processing (WCSP), 1-6 (2013)

7. Sang-Rim Lee, Sung-Hyun Moon, and Inkyu Lee, Downlink distributed antenna systems: Optimal beamforming designs and capacity behavior, In Proc. of 2012 IEEE International Conference on Communications (ICC), 2151-2155 (2012)

8. Thantip Thaikerd and Wiroonsak Santipach, "On optimizing transmit antenna placement for downlink distributed antenna systems with zero-forcing beamforming," In Proc. of 2015 12th International Conference on Electrical Engineering/Electronics, Computer, Telecommunications and Information Technology, 1-5 (2015)

9. Meiyan Ju, Yuheng Li, Wenjie Peng, and Guoping Tan, System outage probability analysis of distributed antenna systems with beamforming, In Proc. of 10th International Conference on Wireless Communications, Networking and Mobile Computing (WiCOM), 264-268 (2014)

10. R. N. McDonough and A. D. Whalen, Detection of signals in noise, 2nd Edition, Academic Press, California, 105-150 (1995)

11. Mai $\mathrm{Vu}$, MISO capacity with per-antenna power constraint, IEEE Transactions on Communications, 59, 5, 1268-1274 (2011)

12. Gordon L. Stuber, Principles of mobile communication, 2th Edition, Kluwer Academic Publishers, New York, Boston, Dordrecht, London, Moscow (2011)

13. Milton Abramowitz and Irene A. Stegun, Handbook of mathematical functions with formulas, graphs, and mathematical tables, 9th Edition, Dover Publications, New York, 890-890 (1970)

14. R. L. Burden and J. D. Faires, Numerical analysis, 4th Edition, PWS KENT Publishing Company, Boston (1989)

15. Wen-jie Peng, Yue-heng Li, Mei-yan Ju, Ping Huang, Guo-ping Tan, Downlink outage probability analysis of distributed MIMO systems over a composite fading channel, Journal on Communications, 35, 6, 161-168 (2014) 